\title{
An Allometric Algorithm for Fractal-Based Cobb-Douglas Function of Geographical Systems
}

\author{
Hongyu Luo ${ }^{1}$ and Yanguang Chen ${ }^{2}$ \\ ${ }^{1}$ Land Requisition Affairs Center of Jilin Province, Changchun 130042, China \\ ${ }^{2}$ Department of Geography, College of Urban and Environmental Sciences, Peking University, Beijing 100871, China \\ Correspondence should be addressed to Yanguang Chen; chenyg@pku.edu.cn
}

Received 2 April 2014; Revised 1 August 2014; Accepted 19 August 2014; Published 20 October 2014

Academic Editor: Nikos I. Karachalios

Copyright (c) $2014 \mathrm{H}$. Luo and Y. Chen. This is an open access article distributed under the Creative Commons Attribution License, which permits unrestricted use, distribution, and reproduction in any medium, provided the original work is properly cited.

\begin{abstract}
The generalized Cobb-Douglas production function has been derived from a general input-output relation based on fractality assumptions. It was proved to be a useful self-affine model for geographical analysis. However, the ordinary least square calculation is always an ineffectual method for the Cobb-Douglas modeling because of the multicollinearity in the logarithmic linear regression. In this paper, a novel approach is proposed to build the geographical Cobb-Douglas models. Combining the concept of allometric scaling with the linear regression technique, we obtain a simple algorithm that can be employed to estimate the parameters of the Cobb-Douglas function. As a case, the algorithm and models are applied to the public transportation of China's cities, and the results validate the allometric algorithm. A conclusion can be drawn that the allometric analysis is an effective way of modeling geographical systems with the general Cobb-Douglas function. This study is significant for integrating the notions of allometry, fractals, and scaling into a new framework to form a quantitative methodology of spatial analysis.
\end{abstract}

\section{Introduction}

The well-known production function was proposed by Cobb and Douglas [1] and consolidated by many economists. Today, the Cobb-Douglas function was proved to be a tool of geographical analysis because geographical systems are always associated with economic systems [2,3]. In fact, many geographical theories such as central place theory came from economics. By geographical assumptions, the generalized Cobb-Douglas production model can be derived in a simple way by using the ideas from fractals and scaling. Thus the fractal concept and the production function were combined to form a new model. This model may be useful for geographical information analysis in the future. Fractals are everywhere [4-6], and fractal dimension is related to information entropy [7]. Fractal geometry can be employed to reveal spatial information of geographical systems, especially, cities as systems and systems of cities [8-25]. What is more, the general Cobb-Douglas function can be applied to modeling natural systems, for example, ecological systems [26].

A good mathematical model is always based on an effective algorithm. However, the ordinary least square (OLS) method cannot be used to estimate the parameter values of the fractal-based Cobb-Douglas model in many cases because of logarithmic multicollinearity. In other words, there are allometric scaling relationships between any two independent variables, which are employed to make models. On the other hand, the allometric scaling is one of the basic laws in geography. It can be utilized to make spatial analyses for many geographical systems $[2,10,27-35]$. The key to solving the problem of the Cobb-Douglas modeling rests with the allometry. In this paper, the allometric analysis is adopted to develop a new approach to evaluating the parameters of the general production function. The other parts are organized as follows. In Section 2, the allometric scaling is demonstrated to be a way of estimating the parameters of the Cobb-Douglas model. In Section 3, the model based on the allometric algorithm is applied to the public transportation of Chinese cities, and the empirical results validate the theoretical derivation. Finally, in Section 4, the related questions are discussed, and the paper is concluded with several pieces of comments.

\section{Mathematical Models}

2.1. New Form of the Production Function. In geography as well as economics, the mathematical models similar to the 
Cobb-Douglas production functions are widely employed to describe the relationship of one output with many inputs. Suppose that there are $m$ elements $X_{i}$ in a geographical system, and each element affects the output $Y$ to some extent $(i=1,2, \ldots, m)$. An assumption can be made as follows:the geographical system is a fractal system in a broad sense. That is, the relationships between the inputs represented by elements $X_{i}$ and the output $Y$ satisfy some self-similar or selfaffine conditions [2]. Because all the elements contribute to the output of the geographical system, the output as response to the elements can be generally expressed as follows:

$$
Y=k f\left(X_{1}, X_{2}, \ldots, X_{m}\right)
$$

in which $k$ is a proportionality coefficient. Taking the complete differential of (1) yields

$$
\mathrm{d} Y=A \sum_{i=1}^{m} \frac{\partial f}{\partial X_{i}} \mathrm{~d} X_{i}=\sum_{i=1}^{m} \frac{\partial Y}{\partial X_{i}} \mathrm{~d} X_{i}
$$

This result is easy to understand by the knowledge of complete differential in higher mathematics. Dividing (2) on both sides with $Y$ gives

$$
\frac{\mathrm{d} Y}{Y}=\sum_{i=1}^{m}\left[\left(\frac{\partial Y}{\partial X_{i}} \frac{X_{i}}{Y}\right) \frac{\mathrm{d} X_{i}}{X_{i}}\right]=\sum_{i=1}^{m} \sigma_{i} \frac{\mathrm{d} X_{i}}{X_{i}} .
$$

The precondition of (3) is that the system depicted by (1) is of fractal structure. If and only if the system is of self-similar or self-affine structure, the parameter $\sigma_{i}$ will be constant. According to our assumption aforementioned, the fractal parameter $\sigma_{i}$ can be defined by

$$
\sigma_{i}=\frac{\partial Y}{\partial X_{i}} \frac{X_{i}}{Y}=\frac{\partial Y / Y}{\partial X_{i} / X_{i}}=\frac{\partial \ln Y}{\partial \ln X_{i}} .
$$

This parameter is in fact an elastic coefficient. Apparently, (4) reflects a partial scaling relation, just indicating that the output $Y$ depends to scale on the element $X_{i}$ regardless of other elements. Equation (3) can be equivalently expressed as

$$
\mathrm{d} \ln Y=\sum_{i=1}^{m} \sigma_{i} \mathrm{~d} \ln X_{i}
$$

Then taking integral on both sides of (5) yields

$$
\int \mathrm{d} \ln Y=\int \sum_{i=1}^{m} \sigma_{i} \mathrm{~d} \ln X_{i}=\sum_{i=1}^{m} \int \mathrm{d} \ln X_{i}^{\sigma_{i}} .
$$

Apparently, from (6) it follows that

$$
\ln Y=\sum_{i=1}^{m} \ln X_{i}^{\sigma_{i}}+C,
$$

where $C$ refers to the integral constant. A simple mathematical transform of (7) yields

$$
Y=K \prod_{i=1}^{m} X_{i}^{\sigma_{i}}
$$

which is just the generalized Cobb-Douglas function indicative of self-affine patterns $[2,3]$. In the function, the constant $K=\exp C$ refers to the output coefficient. If $m=2$ as given, then (8) can be reduced to the common Cobb-Douglas function

$$
Y=K X_{1}^{\sigma_{1}} X_{2}^{\sigma_{2}}
$$

where $X_{1}$ and $X_{2}$ represent capital and labor, respectively. In a city system, $X_{1}$ and $X_{2}$ can represent population size and land-use area.

The traditional production function is a special case of the generalized production function. It is clear that (8) denotes a complete relation, while (4) represents a partial relation. In this case, if (8) is true, we cannot integrate (4) over $X_{i}$. Otherwise a contradiction will take place if we take integral of (4). Now suppose that only $X_{i}$ influences $Y$ with other elements being invariable. Then the equation $\mathrm{d} \ln Y=\sigma_{i} \mathrm{~d} \ln X_{i}$ can be derived from (4), and integrating this relation gives the following power function:

$$
Y=C_{i} X_{i}^{\sigma_{i}}
$$

This is a generalized allometric function [2, 36-38]. There is a logical contradiction between (8) and (10). Comparing (10) with (8) shows why (4) cannot be calculated the integral when (10) holds. Evidently, we can get a multivariate linear regression equation by taking logarithms on both sides of (8); in the same way, a univariate linear regression equation can be gotten by taking logs of (10). According to the knowledge of statistics related, if a response variable depends on many explanatory variables, but only one of which is considered, then the estimation results of the parameters of the regression equation will greatly deviate from the true values. In short, (10) will be true if and only if others elements $X_{j}(j \neq i)$ are all fixed.

A general allometric scaling relation can be derived from the generalized Cobb-Douglas function. Taking two elements, say, $X_{i}$ and $X_{j}$, into consideration in terms of (10), we have

$$
X_{i} \propto X_{j}^{\sigma_{j} / \sigma_{i}}
$$

This is the well-known allometric relation of any two elements $[28,39]$. Suppose the dimension of $X_{i}$ is $D_{i}$, and the dimension of $X_{j}$ is $D_{j}$. According to the principle of dimensional homogeneity, we have

$$
X_{i} \propto X_{j}^{D_{i} / D_{j}}
$$

The principle of dimensional homogeneity is also called the "principle of dimension consistency" [29], which comes from the proportion axiom in mathematics. Comparing (12) with (11) yields a fractal parameter relation such as [2]

$$
\sigma_{i} D_{i}=\sigma_{j} D_{j}
$$

which suggests that the ratio of two fractal dimensions equals the ratio of two elastic coefficients, but a fractal dimension is 
reciprocally proportional to its corresponding elastic coefficient. Substituting (11) or (12) into (8) repeatedly yields

$$
Y=c_{i} X_{i}^{d_{i}}
$$

where $c_{i}$ is a proportionality coefficient and $d_{i}$ is a generalized allometric scaling exponent. Clearly, the scaling exponent $d_{i}$ is relative to the fractal dimension $D_{i}$ and $D_{j}$. Equation (14) shares the same form as (10), but they have different physical meaning. Equation (10) is true if and only if the final output depends only on one element, whereas (14) is true when the final output depends on many elements, between which there exist allometric scaling relations. However, if the allometric relation appears between any pair of urban elements, the parameters of the log-linear expression of (8) cannot be estimated by using the OLS method. Otherwise, we will inevitably come across the problem of multicollinearity. Thus we need a new algorithm for the Cobb-Douglas modeling of geographical systems.

2.2. Allometric Algorithm. The fractal-based Cobb-Douglas equation can be formally solved by the OLS algorithm, but the results may be unacceptable. Taking natural logarithm on both sides of (8) gives

$$
\ln Y=\ln K+\sigma_{1} \ln X_{1}+\sigma_{2} \ln X_{2}+\cdots+\sigma_{m} \ln X_{m},
$$

which is clearly a multivariable linear equation. It seems as if the technique of multivariate linear regression can be employed to estimate the values of model parameters. However, the independent variables are always related to one another in practice so that the parameters cannot be properly evaluated because of multicollinearity. A discovery is that the allometric analysis can be used to solve the generalized CobbDouglas equations. Let $i=1,2, \ldots, m$. Then multiplying (14) by (14) again and again yields

$$
Y=\sqrt[m]{c_{1} c_{2} \cdots c_{m}} X_{1}^{d_{1} / m} X_{2}^{d_{2} / m} \cdots X_{m}^{d_{m} / m}
$$

This suggests a partial scaling relation. In fact, multiplying $X_{i}$ by a scale factor $\lambda$ gives

$$
Y\left(\lambda X_{i}\right)=\lambda^{\sigma_{i}} Y\left(X_{i}\right)
$$

which suggest a self-affine fractal structure [3]. Comparing (16) with (8) shows

$$
\sigma_{i}=\frac{d_{i}}{m}
$$

An allometric algorithm can be presented for solving the generalized production model. In fact, (14) and (16) indicate a new approach to estimate model parameters, and (18) suggests a simple method of evaluating the partial scaling exponents. In short, by means of the allometric scaling relations between each independent variable and the dependent variable, we can replace the multiple log-linear regression with a set of simple log-linear regressions to estimate the model parameters and then build the Cobb-Douglas model.
2.3. Fractal Meaning of Model Parameters. It is necessary to demonstrate that the parameters of the Cobb-Douglas model are associated with fractal dimension. The Hausdorff dimension has been proved to be equivalent to Shannon's information entropy [7]. Revealing the dimension meaning of the model parameters is helpful for spatial analysis of geographical systems. The key to understanding the property of fractional dimension of the Cobb-Douglas model parameters lies in the geometric measure relation, which is also termed fractal measure relation [40]. Suppose that $D_{Y}$ denotes the dimension of $Y$. Comparing (14) with the geometric measure relation based on the principle of dimensional consistency

$$
Y \propto X_{i}^{D_{Y} / D_{i}}
$$

we have

$$
d_{i}=\frac{D_{Y}}{D_{i}}=m \sigma_{i} .
$$

This suggests that the parameter $d_{i}$ has fractal dimension property in a broad sense. In fact, the generalized allometric scaling exponent is a ratio of the dimension of the output variable to that of an input variable. It is obvious that (12) can be derived from (19). Thus a generalized fractal dimension equation can be obtained as follows:

$$
D_{Y}=d_{i} D_{i}=d_{j} D_{j}=m \sigma_{i} D_{i}=m \sigma_{j} D_{j},
$$

which gives the mathematical relationships between different fractal dimensions, different elastic coefficients, and different allometric scaling exponents.

The fractal parameter equations are revealing for us the idea to link the generalized Cobb-Douglas function with allometric analysis. Although the scaling exponent in (16) may not be equivalent to the corresponding parameters in (8), the ratio of these parameters equals each other. For example, considering two elements $X_{i}$ and $X_{j}$, we have

$$
\frac{d_{i}}{d_{j}}=\frac{\sigma_{i}}{\sigma_{j}},
$$

which suggests that the ratio of two allometric scaling exponents equals the ratio of two elastic coefficients, but an allometric scaling exponent is be directly proportional to its corresponding elastic coefficient. This relation is important for fractal study of geographical systems. In fact, when we make an allometric analysis of different components in a system, what we are really concerned with is the ratio of two fractal dimensions instead of each fractal dimension $[3,28]$. Therefore, we can explore the ratio of dimensions by substituting (14) for (8). That is, a fractal study can be made from allometric analysis to the Cobb-Douglas modeling.

The main points of the mathematical process can be summarized as follows [3]. First, the Cobb-Douglas function is a fractal model indicating self-affinity. Second, the model parameters are relative to the fractal dimension in a broad sense. Third, the relationships between one element and other elements follow the law of allometric growth. Last but not the least, if the input-output relations of a geographical system can be characterized with the Cobb-Douglas function, we can investigate the system by using only a few variables, which can simplify the analytical process to a great extent. 


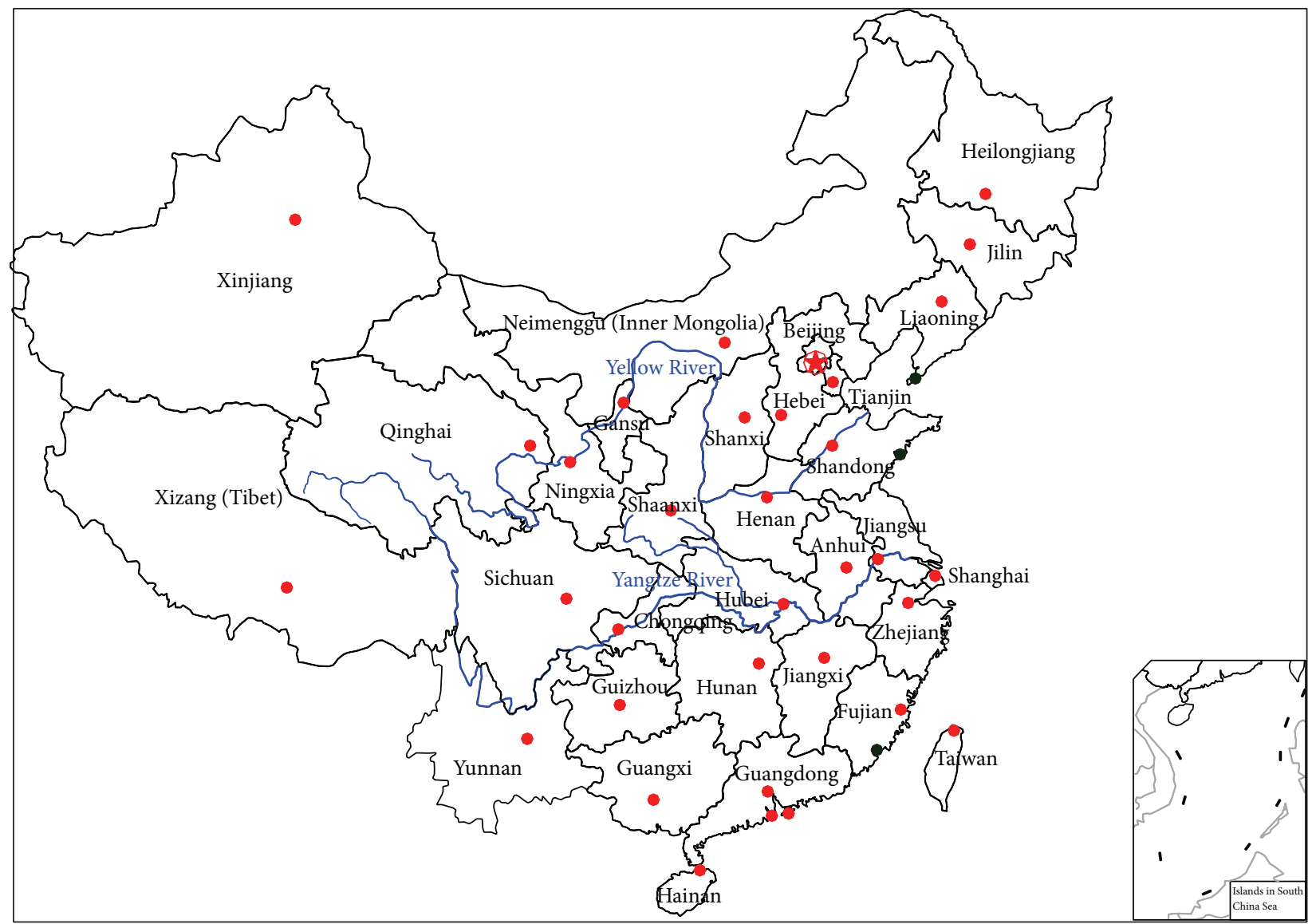

\footnotetext{
National capital

- Provincial capital

- Great port city
}

Figure 1: A sketch map of 31 regions including the 27 provinces, autonomous regions, and 4 municipalities directly under the Central Government of China.

\section{Empirical Analysis}

3.1. Materials and Methods. A case study can be made by applying the general Cobb-Douglas model and allometric algorithm to the public transportation of Chinese cities. The study area includes the whole mainland of China (Figure 1). Urban transportation is one of important aspects of new science of cities [41]. The observational data come from China Statistical Yearbook of National Bureau of Statistics of the People's Republic of China. Three variables are adopted in this statistics as follows: first, the year-end number of public vehicles in operation (unit), which is abbreviated to "vehicle number"; second, the total length of roads in operation $(\mathrm{km})$, which is represented by "road length" for short; third, the volume of passengers transported by public traffic vehicles (10000 person-times), which is abbreviated to "passenger volume." The three variables fall into two types: two input (independent) variables and one output (dependent) variable (Table 1). The public transportation can be divided into two groups: the major group includes bus and trolley bus, and the miner group includes subways, light rail, and streetcar.
Based on these variables, partial basic statistical datasets on the public transportation of China's cities are tabulated by region as follows (Table 2 ).

Using log-linear regression analysis and allometric analysis, we can build two kinds of Cobb-Douglas model for the urban transportation of China. One is for the whole transportation, including the major group and minor group (global analysis); the other is for the major group, including bus and trolley bus (local analysis). Because of the incomplete statistical dataset, we do not make models for the minor group including subway, light rail, and streetcar. First of all, a structural analysis is made; then, a simple dynamical analysis is implemented. All these analyses are based on the crosssectional datasets of the public transportation of China's cities.

3.2. Results. For comparison, let us examine the wrong approach to modeling the urban transportation in the first place. If the multivariable log-linear regression is employed to estimate the model parameters, the results will be deviant. 
TABLE 1: The input variables and output variable for quantitative analysis of the public transportation of China's cities (2006-2012).

\begin{tabular}{lcccc}
\hline Type & Variable & Abbreviation & Measurement unit & Symbol \\
\hline $\begin{array}{l}\text { Input } \\
\text { (independent variables) }\end{array}$ & Year-end number of public vehicles in operation & Vehicle number & unit & $x_{1}$ \\
Output & Total length of roads in operation & Road length & km & $x_{2}$ \\
\hline \begin{tabular}{l} 
(dependent variable) \\
\hline
\end{tabular} & Volume of passengers transported by public traffic vehicles & Passenger volume & 10000 person-times & $y$ \\
\hline
\end{tabular}

TABLE 2: Basic datasets on the public transportation in China's cities by region (2012).

\begin{tabular}{|c|c|c|c|c|c|c|}
\hline \multirow{2}{*}{ Region } & \multicolumn{3}{|c|}{ Global datasets (public transportation) } & \multicolumn{3}{|c|}{ Local datasets (bus and trolley bus) } \\
\hline & Vehicle number & Road length & Passenger volume & Vehicle number & Road length & Passenger volume \\
\hline Anhui & 11992.0 & 10535.0 & 212719.0 & 11992.0 & 10535.0 & 212719.0 \\
\hline Beijing & 25831.0 & 19989.0 & 761578.0 & 22146.0 & 19547.0 & 515416.0 \\
\hline Chongqing & 8540.0 & 8959.0 & 201331.0 & 7982.0 & 8828.0 & 176968.0 \\
\hline Fujian & 11823.0 & 15627.0 & 224703.0 & 11823.0 & 15627.0 & 224703.0 \\
\hline Gansu & 5214.0 & 4907.0 & 102846.0 & 5214.0 & 4907.0 & 102846.0 \\
\hline Guangdong & 53089.0 & 87797.0 & 1003098.0 & 50729.0 & 87384.0 & 739359.0 \\
\hline Guangxi & 7430.0 & 9323.0 & 142505.0 & 7430.0 & 9323.0 & 142505.0 \\
\hline Guizhou & 5031.0 & 5305.0 & 132200.0 & 5031.0 & 5305.0 & 132200.0 \\
\hline Hainan & 2614.0 & 5600.0 & 43306.0 & 2614.0 & 5600.0 & 43306.0 \\
\hline Hebei & 16493.0 & 18812.0 & 203954.0 & 16493.0 & 18812.0 & 203954.0 \\
\hline Heilongjiang & 14364.0 & 15087.0 & 223956.0 & 14364.0 & 15087.0 & 223956.0 \\
\hline Henan & 18137.0 & 18337.0 & 263718.0 & 18137.0 & 18337.0 & 263718.0 \\
\hline Hubei & 16982.0 & 17354.1 & 338901.0 & 16670.0 & 17298.0 & 330613.0 \\
\hline Hunan & 13148.0 & 14132.0 & 272165.0 & 13148.0 & 14132.0 & 272165.0 \\
\hline Inner Mongolia & 5586.0 & 10650.0 & 96349.0 & 5586.0 & 10650.0 & 96349.0 \\
\hline Jiangsu & 30956.0 & 49903.2 & 470233.0 & 30380.0 & 49793.0 & 427578.0 \\
\hline Jiangxi & 7852.0 & 11648.0 & 127961.0 & 7852.0 & 11648.0 & 127961.0 \\
\hline Jilin & 10912.0 & 11254.5 & 170561.0 & 10532.0 & 11200.0 & 165336.0 \\
\hline Liaoning & 20968.0 & 21520.8 & 428367.0 & 20500.0 & 21384.0 & 401457.0 \\
\hline Ningxia & 3042.0 & 4813.0 & 37441.0 & 3042.0 & 4813.0 & 37441.0 \\
\hline Qinghai & 2067.0 & 1937.0 & 39135.0 & 2067.0 & 1937.0 & 39135.0 \\
\hline Shaanxi & 10948.0 & 9206.9 & 254599.0 & 10840.0 & 9187.0 & 248687.0 \\
\hline Shandong & 32869.0 & 44682.0 & 398268.0 & 32869.0 & 44682.0 & 398268.0 \\
\hline Shanghai & 19825.0 & 23658.2 & 507933.0 & 16695.0 & 23190.0 & 280360.0 \\
\hline Shanxi & 7851.0 & 13369.0 & 124838.0 & 7851.0 & 13369.0 & 124838.0 \\
\hline Sichuan & 19628.0 & 19179.5 & 357333.0 & 19388.0 & 19140.0 & 347025.0 \\
\hline Tianjin & 9031.0 & 12870.7 & 129951.0 & 8405.0 & 12732.0 & 118721.0 \\
\hline Tibet & 396.0 & 834.0 & 7139.0 & 396.0 & 834.0 & 7139.0 \\
\hline Xinjiang & 8155.0 & 7568.0 & 151397.0 & 8155.0 & 7568.0 & 151397.0 \\
\hline Yunnan & 8187.0 & 16329.0 & 148409.0 & 8187.0 & 16329.0 & 148409.0 \\
\hline Zhejiang & 23060.0 & 40606.0 & 311024.0 & 22892.0 & 40558.0 & 310463.0 \\
\hline
\end{tabular}

Source: [43].

For the global modeling (public transportation), the mathematical expression is as follows:

$$
\widehat{y}=26.9052 x_{1}^{1.3306} x_{2}^{-0.3663},
$$

where $x_{1}$ refers to vehicle number, $x_{2}$ refers to road length, and $y$-hat refers to passenger volume. The hat symbol “へ” indicates "predicted value." For the local modeling (bus and trolley bus), the model is

$$
\widehat{y}=36.7876 x_{1}^{1.2959} x_{2}^{-0.3690},
$$

in which the notation is the same as in (23). The main statistics for these models are displayed in Table 3. Obviously, based on the statistics, the levels of confidence of the two models are both greater than $99 \%$. However, the scaling exponents are abnormal because the elasticity of $x_{2}$ versus $y$ is minus. If the models are true, this will suggest that the length of roads makes a negative contribution towards the traffic volume of passengers. This case cannot take place in the real world. The deviant parameter values mean that the multivariable linear regression is improper and it is necessary to adopt a valid approach to evaluating model parameters.

In contrast, if we employ the allometric analysis based on the simple linear regression to estimate model parameters, the result will be different and convincing. The relationship 
TABLE 3: Main statistics based on the multiple linear regressions for two types of models on the public transportation of China's cities (2012).

\begin{tabular}{|c|c|c|c|c|c|}
\hline \multicolumn{2}{|l|}{ Item } & \multicolumn{2}{|c|}{ Global modeling (public transportation) } & \multicolumn{2}{|c|}{ Local modeling (bus and trolley bus) } \\
\hline Type & Object & Statistic & $P$ values (sig.) & Statistic & $P$ values (sig.) \\
\hline Multiple $R^{2}$ & Model & 0.9606 & - & 0.9703 & - \\
\hline Standard error $s$ & Model & 0.2009 & - & 0.1642 & - \\
\hline$F$ statistics & Model & 341.5719 & $2.1518 * 10^{-20}$ & 457.8210 & $4.1012 * 10^{-22}$ \\
\hline \multirow{3}{*}{ Parameter and statistic } & Constant & 8.6391 & $2.1902 * 10^{-9}$ & 11.5756 & $3.4669 * 10^{-12}$ \\
\hline & $x_{1}$ & 10.5482 & $2.9246 * 10^{-11}$ & 12.3132 & $8.0917 * 10^{-13}$ \\
\hline & $x_{2}$ & -2.7743 & 0.0097 & -3.3900 & 0.0021 \\
\hline
\end{tabular}

The standard error $s$ is for the linear regression prediction; $P$ value or sig. implies the corresponding significance of $F$ and $t$ statistics, that is, a kind of probability for null hypothesis.

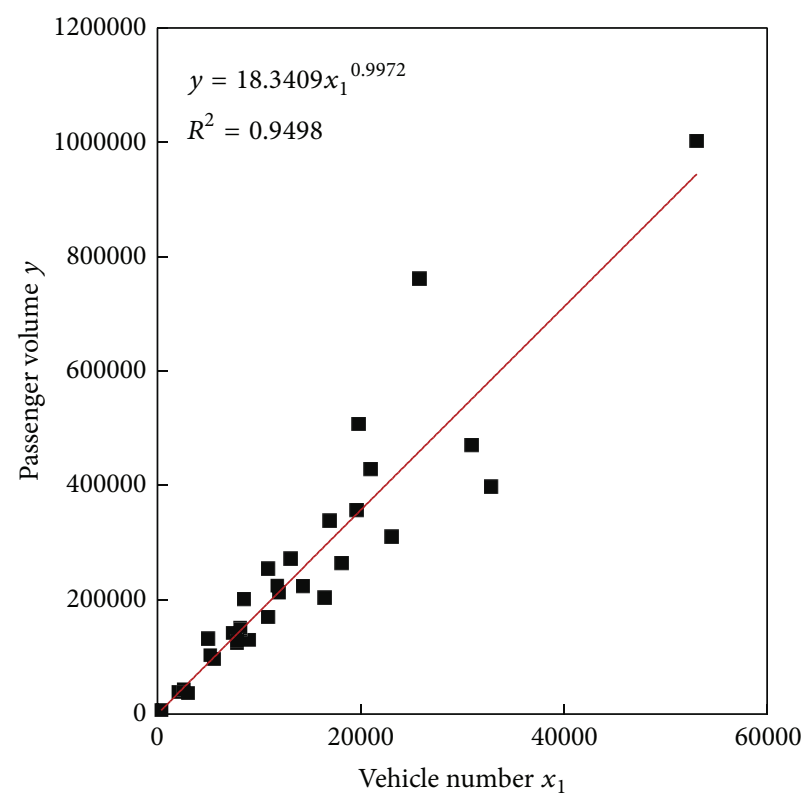

(a) Vehicles and passengers

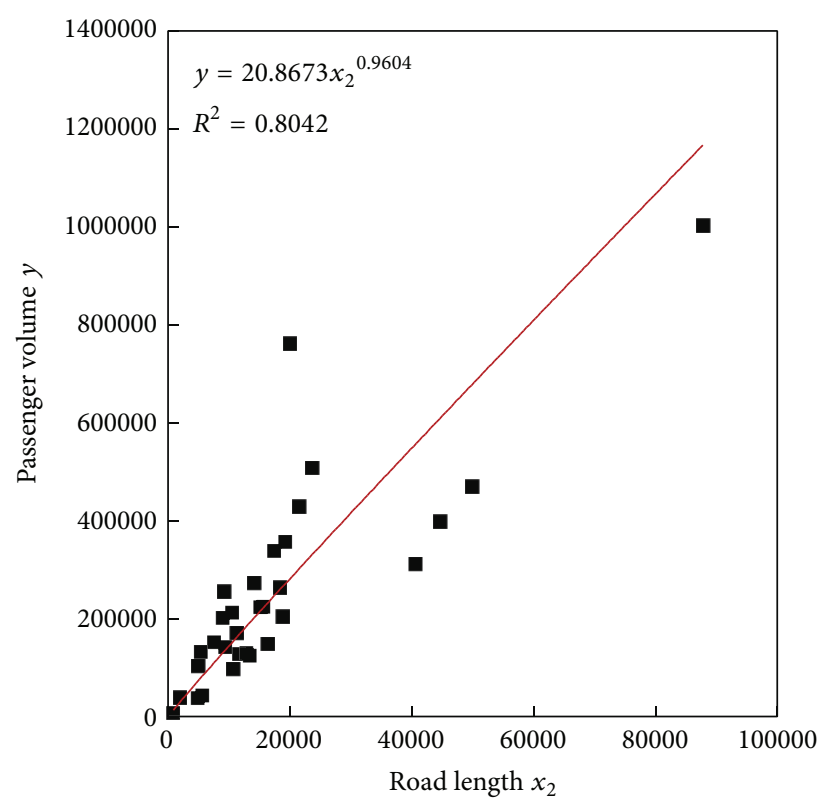

(b) Roads and passengers

FIGURE 2: The allometric scaling relations between vehicles/roads and passengers: global modeling of the urban public transportation of China (2012).

between vehicle number and road length as well as passenger volume follows the law of allometric scaling (Figures 2 and 3). This is a kind of cross-sectional allometric growth [28]. Using (14) to make log-linear regression analyses, we can estimate the parameter values of the Cobb-Douglas function easily. According to the mathematical process from (14) to (16), two allometric models can be integrated into a Cobb-Douglas model. The first step is to calculate the allometric scaling exponents, and the second step is to evaluate the parameters of the general production function. For the global modeling (public transportation), the allometric models are as follows:

$$
\begin{aligned}
& \widehat{y}=18.3409 x_{1}^{0.9972}, \\
& \widehat{y}=20.8673 x_{2}^{0.9604} .
\end{aligned}
$$

The values of the goodness of fit are $R^{2}=0.9498$ and $R^{2}=$ 0.8042 , respectively (Figure 2). Multiplying (25) by (26) yields the following global modeling result:

$$
\widehat{y}=19.5633 x_{1}^{0.4986} x_{2}^{0.4802} \text {. }
$$

For the local modeling (bus and trolley bus), the allometric models are as follows:

$$
\begin{aligned}
& \widehat{y}=25.8559 x_{1}^{0.9558}, \\
& \widehat{y}=32.1484 x_{2}^{0.9088} .
\end{aligned}
$$

The determination coefficient values are $R^{2}=0.9581$ and $R^{2}=0.8097$, respectively (Figure 3 ). Multiplying (28) by (29) gives the local modeling result in the following form:

$$
\widehat{y}=28.8310 x_{1}^{0.4779} x_{2}^{0.4544} \text {. }
$$




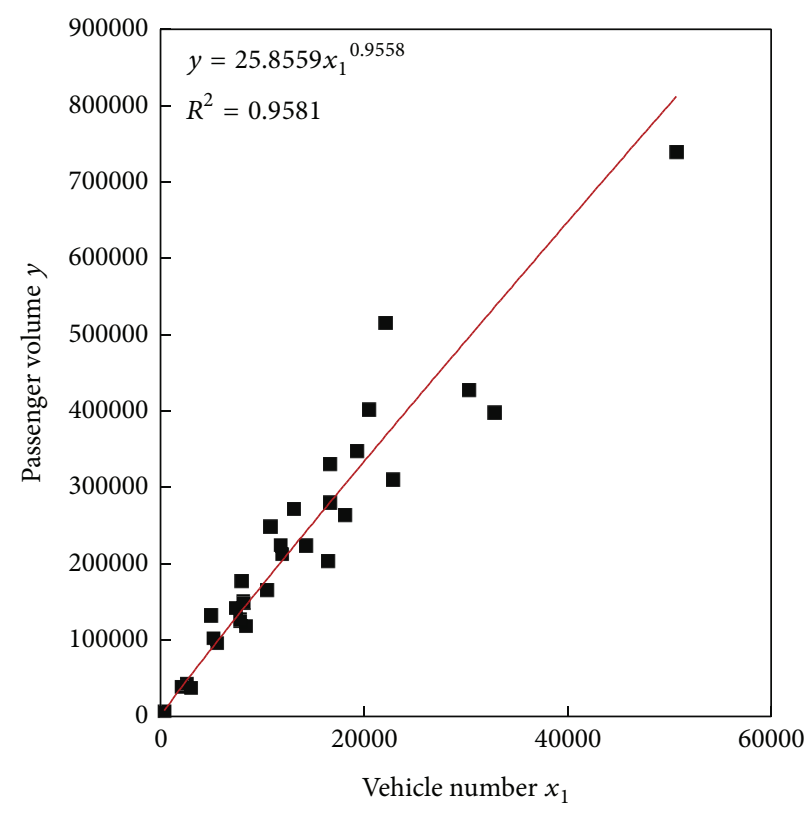

(a) Vehicles and passengers

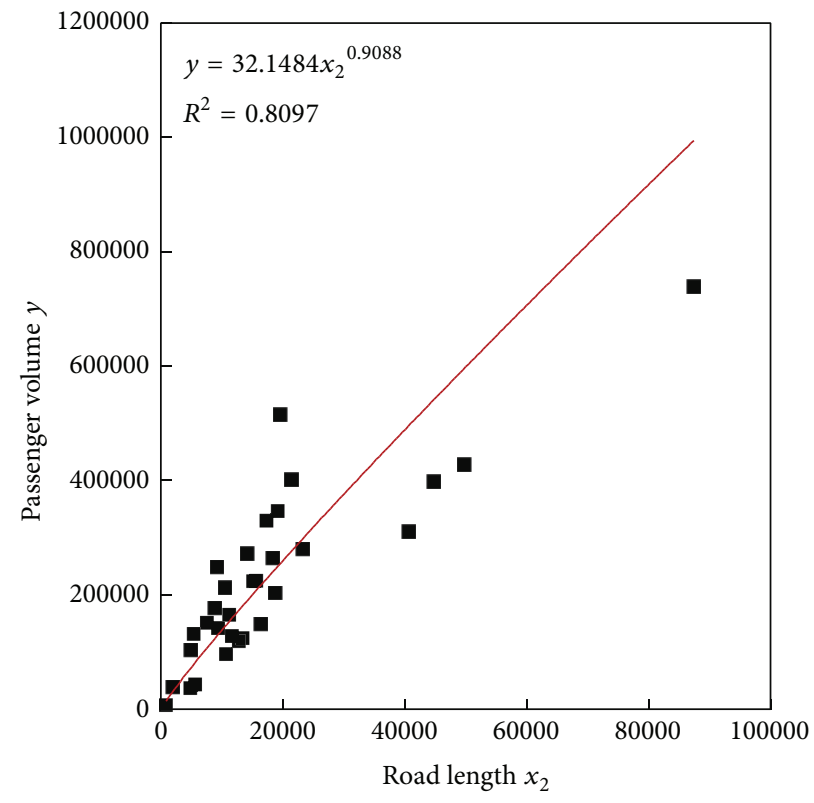

(b) Roads and passengers

FIGURE 3: The allometric scaling relations between vehicles/roads and passengers: local modeling of the urban public transportation of China (2012).

TABLE 4: Parameters and main statistics based on allometric scaling for two types of models on public transportation of China's cities (2012).

\begin{tabular}{lcccc}
\hline \multirow{2}{*}{ Parameter and statistic } & \multicolumn{2}{c}{ Global modeling (public transportation) } & \multicolumn{3}{c}{ Local modeling (bus and trolley bus) } \\
& $x_{1}$ versus $y$ & $x_{2}$ versus $y$ & $x_{1}$ versus $y$ & 25.8559 \\
$x_{2}$ versus $y$ \\
\hline Proportionality coefficient $c$ & 18.3409 & 20.8673 & 0.9558 & 32.1484 \\
Scaling exponent $d$ & 0.9972 & 0.9604 & 0.9581 & 0.9088 \\
Determination coefficient $R^{2}$ & 0.4986 & 0.8042 & 0.4779 & 0.8097 \\
Cross elastic coefficient $\sigma$ & & 0.4802 & & 0.4544 \\
\hline
\end{tabular}

So far, we have finished the process of mathematical modeling from the allometric scaling relations to the CobbDouglas function. The two models, (27) and (30), are fair and reasonable and thus acceptable. In the models, a proportionality coefficient of the Cobb-Douglas model is the geometrical average of allometric proportionality coefficients, and each cross elastic coefficient is the corresponding allometric scaling exponent divided by the number of independent variables (Table 4 ).

Before using the parameter values to explain the system of China's public urban transportation, we clarify the relationships between the parameters and the geographical system. Generally speaking, it is a measurement that makes a link between mathematical models and empirical phenomena [42]. In theory, each system can be made a measurement, and thus we have one or more measure values (e.g., length, area, size, density); each measure corresponds to a dimension, and thus we have one or more dimension values (e.g., 1, 2, 3, 1.262, 1.585). If a system has a characteristic scale, the dimension will be an integer $(0,1,2,3)$ and will give no useful information, and we can use the measure values to make an analysis; on the contrary, if a system has no characteristic scale, the measure values will be uncertain, and we can use the dimension values to make an analysis [2]. In the latter case, the dimension will be a fractional value instead of an integral number. In this case study, the spatial measures that we employ are vehicle number, road length, and passenger volume, as indicated above. Because human geographical systems bear no characteristic scale, we had better utilize fractal dimensions or scaling exponents based on these measures to make analyses. The relationships between the scaling exponents, elastic coefficient, and fractal dimension are shown by (13), (20), (21), and (22), which have been explained above. The best approach to understanding the mathematical models and parameters is the mathematical process. According to these equations, we can understand the fractal parameters, and using these fractal parameters, especially the scaling exponents, we can research into a geographical system.

In terms of the modeling processes and the scaling exponent values, we can reveal the geographical features of public transportation of China's cities. First, the system of public urban transportation follows the allometric scaling law. Thus the system can be described with generalized fractal dimension. As far as the case in 2012 is concerned, according to the allometric scaling exponent values (Figures 2 and 3), the fractal dimension of passenger volume is greater than that 
TABle 5: Parameters and statistics of allometric models on public transportation of China's cities (2006-2012).

\begin{tabular}{lcccccccc}
\hline Model parameter & & 2006 & 2007 & 2008 & 2009 & 2010 & 2011 & 2012 \\
\hline \multirow{2}{*}{ Vehicle and passenger } & $c$ & 3.8349 & 11.3699 & 42.1560 & 12.1662 & 6.1807 & 20.6520 & 18.3409 \\
& $d$ & 1.1445 & 1.0344 & 0.9083 & 1.0393 & 1.1115 & 0.9841 & 0.9972 \\
& $R^{2}$ & 0.8393 & 0.9137 & 0.7235 & 0.8471 & 0.9240 & 0.9524 & 0.9498 \\
\hline \multirow{2}{*}{ Road and passenger } & $c$ & 1112.9227 & 4890.0248 & 7285.5613 & 3182.1909 & 13.5510 & 21.4487 & 20.8673 \\
& $d$ & 0.5763 & 0.4150 & 0.3908 & 0.4609 & 1.0025 & 0.9573 & 0.9604 \\
& $R^{2}$ & 0.3358 & 0.3151 & 0.2682 & 0.2322 & 0.8084 & 0.8064 & 0.8042 \\
\hline
\end{tabular}

Before 2006, the datasets are not incomplete; that is, there is no observational data for the total length of roads in operation.

of vehicle number and road length, and the fractal dimension of vehicle number is less than that of road length. The less fractal dimension indicates a larger developing space. This suggests that the volume of passengers depends more on the number of vehicles than on the length of roads, and increasing a unit of vehicles or roads can lead to increasing less than one unit of passengers. Second, the allometric scaling and the Cobb-Douglas relation are all evolutive patterns rather than inherent patterns. From 2010 on, both the vehiclepassenger relation and the road-passenger relation follow the allometric scaling law; thus the datasets can be fitted to the Cobb-Douglas function. However, before 2009, the relationships between the road length and passenger volume did not conform to the law of allometric growth, and the Cobb-Douglas model cannot be built for early public urban transportation (Table 5). What is more, the goodness of fit of the allometric regression modeling has been escalating in a fluctuant way (Figure 4). From the above analyses, two conclusions can be drawn as follows. First, the structure of the public transportation of China's cities has been improved year after year; second, it is more efficient to optimize vehicles than to increase roads of Chinese urban transportation.

\section{Discussion and Conclusions}

The Cobb-Douglas production function comes from economic problems, but it can be applied to many kinds of systems such as city systems and ecosystems $[2,3,26]$. In other words, the Cobb-Douglas function is a universal model, which can be used to describe the input-output relations of natural and social systems in various fields. In this sense, the abovementioned results of derivation can be generalized to other fields, and the precondition is that the systems which will be studied have self-affine fractality. Compared with the previous works [3], the novelty of this study is that a simple allometric algorithm is advanced to estimate the parameters of the Cobb-Douglas models for geographical analysis. The case of public transportation of cities shows the effect of this algorithm. The limitation of this study lies in a lack of dynamical analysis based on time series despite the estimated values of the allometric scaling exponent based on the temporal dimension.

From the theoretical derivation and empirical evidence, the main conclusions of this study can be reached as follows. First, the allometric scaling is associated with the Cobb-Douglas

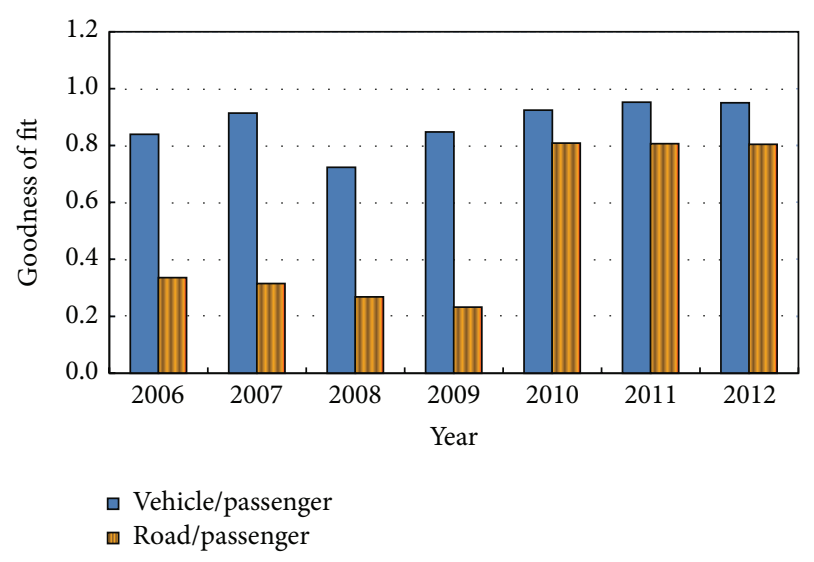

Figure 4: A histogram of the goodness of fit for the allometric scaling relations between vehicle/road and passenger (2006-2012).

function. The cross elastic coefficients of the generalized production function are correlated with the allometric scaling exponents. The allometric analysis provides an efficient approach to estimate the parameters of the Cobb-Douglas model. Second, both fractal dimension and scaling exponent are important indexes for the geographical analysis using the Cobb-Douglas function. An allometric scaling exponent is a ratio of generalized fractal parameters. If the fractal dimension cannot be directly evaluated for a geographical system, the scaling exponents can be used as substitutes. Third, fractal geometry, allometric scaling, and the production function can be integrated into a new framework. Based on this framework, we can develop an effective method for spatial analysis of geographical systems. It is hard to explain every question in a few lines of words. Due to limited space, the related problems remain to be solved in future studies.

\section{Conflict of Interests}

The authors declare that there is no conflict of interests regarding the publication of this paper.

\section{Acknowledgments}

This research was sponsored by the National Natural Science Foundation of China (Grant no. 41171129). The support is 
gratefully acknowledged. The authors would like to thank the anonymous reviewers whose constructive comments were helpful in improving the quality of this paper.

\section{References}

[1] C. W. Cobb and P. H. Douglas, "A theory of production," American Economic Review, vol. 18, no. 1, pp. 139-165, 1928.

[2] Y. G. Chen, Fractal Urban System: Scaling, Symmetry, and Spatial Complexity, Scientific Press, Beijing, China, 2008, (Chinese).

[3] Y. G. Chen and J. Y. Lin, "Modeling the self-affine structure and optimization conditions of city systems using the idea from fractals," Chaos, Solitons and Fractals, vol. 41, no. 2, pp. 615-629, 2009.

[4] M. F. Barnsley, Fractal Everywhere, Academic Press, San Diego, Calif, USA, 1988.

[5] M. Batty, "The size, scale, and shape of cities", Science, vol. 319, no. 5864, pp. 769-771, 2008.

[6] B. B. Mandelbrot, The Fractal Geometry of Nature, W. H. Freeman, New York, NY, USA, 1983.

[7] B. Y. Ryabko, "Noise-free coding of combinatorial sources, Hausdorff dimension and Kolmogorov complexity," Problemy Peredachi Informatsii, vol. 22, no. 3, pp. 16-26, 1986.

[8] M. Batty, Cities and Complexity: Understanding Cities with Cellular Automata, Agent-Based Models, and Fractals, The MIT Press, London, UK, 2005.

[9] M. Batty and P. A. Longley, "The morphology of urban land use," Environment and Planning B: Planning and Design, vol. 15, no. 4, pp. 461-488, 1988.

[10] M. Batty and P. A. Longley, Fractal Cities: A Geometry of Form and Function, Academic Press, London, UK, 1994.

[11] L. Benguigui, D. Czamanski, M. Marinov, and Y. Portugali, "When and where is a city fractal?" Environment and Planning B: Planning and Design, vol. 27, no. 4, pp. 507-519, 2000.

[12] Y. G. Chen, "Fractal dimension evolution and spatial replacement dynamics of urban growth," Chaos, Solitons \& Fractals, vol. 45, no. 2, pp. 115-124, 2012.

[13] Y. G. Chen and J. J. Wang, "Multifractal characterization of urban form and growth: the case of Beijing," Environment and Planning B: Planning and Design, vol. 40, no. 5, pp. 884-904, 2013.

[14] P. Frankhauser, "Aspects fractals des structures urbaines," $L$ ' Espace Géographique, vol. 19, no. 1, pp. 45-69, 1990.

[15] P. Frankhauser, The Fractal Aspects of Urban Structures, Economica, Paris, France, 1994, (French).

[16] P. Frankhauser, "The fractal approach: a new tool for the spatial analysis of urban agglomerations," Population, vol. 10, no. 1, pp. 205-240, 1998

[17] J. Feng and Y. G. Chen, "Spatiotemporal evolution of urban form and land-use structure in Hangzhou, China: evidence from fractals," Environment and Planning B: Planning and Design, vol. 37, no. 5, pp. 838-856, 2010.

[18] J. S. Liu and Y. G. Chen, "A fractal study on the spatial structure of systems of towns in Northeast China," Scientia Geographica Sinica, vol. 15, no. 2, pp. 136-143, 1995.

[19] J. S. Liu and Y. G. Chen, "Researches on the fractional dimensions of the spatial structure of the urban system as fractals in Northeast China," Human Geography, vol. 15, no. 6, pp. 9-16, 2000, (Chinese).
[20] J. S. Liu and Y. G. Chen, "Multifractal measures based on manland relationships of the spatial structure of the urban system in Henan," Scientia Geographica Sinica, vol. 23, no. 6, pp. 713-720, 2003, (Chinese).

[21] H. Y. Luo and Y. G. Chen, "On the methods of characterizing urban land-use form using fractal dimension," Journal of Northeast Normal University (Natural Science Edition), vol. 34, no. 4, pp. 107-113, 2002, (Chinese).

[22] I. Thomas, P. Frankhauser, and M. L. de Keersmaecker, "Fractal dimension versus density of built-up surfaces in the periphery of Brussels," Papers in Regional Science, vol. 86, no. 2, pp. 287308, 2007.

[23] I. Thomas, P. Frankhauser, and C. Biernacki, “The morphology of built-up landscapes in Wallonia (Belgium): a classification using fractal indices," Landscape and Urban Planning, vol. 84, no. 2, pp. 99-115, 2008.

[24] R. White and G. Engelen, "Cellular automata and fractal urban form: a cellular modelling approach to the evolution of urban land-use patterns," Environment and Planning A, vol. 25, no. 8, pp. 1175-1199, 1993.

[25] R. White and G. Engelen, "Urban systems dynamics and cellular automata: fractal structures between order and chaos," Chaos, Solitons and Fractals, vol. 4, no. 4, pp. 563-583, 1994.

[26] Y. G. Chen, Mathematical Methods for Geography, Scientific Press, Beijing, China, 2011, (Chinese).

[27] M. Batty, R. Carvalho, A. Hudson-Smith, R. Milton, D. Smith, and P. Steadman, "Scaling and allometry in the building geometries of greater London," The European Physical Journal BCondensed Matter and Complex Systems, vol. 63, no. 3, pp. 303314, 2008.

[28] Y. G. Chen, "Characterizing growth and form of fractal cities with allometric scaling exponents," Discrete Dynamics in Nature and Society, vol. 2010, Article ID 194715, 22 pages, 2010.

[29] Y. Lee, "An allometric analysis of the US urban system: 196080," Environment and Planning A, vol. 21, no. 4, pp. 463-476, 1989.

[30] J. S. Liu and Y. G. Chen, "The spatial complexity of the law of allometric growth and urban population density," Journal of Northeast Normal University, vol. 36, no. 4, pp. 139-148, 2004, (Chinese).

[31] J. S. Liu and Y. G. Chen, "An allometric analysis of the Shandong urban system using ideas from fractals," Scientia Geographica Sinica, vol. 25, no. 2, pp. 135-141, 2005, (Chinese).

[32] C. P. Lo and R. Welch, "Chinese urban population estimates," Annals of the Association of American Geographers, vol. 67, no. 2, pp. 246-253, 1977.

[33] P. A. Longley, M. Batty, and J. Shepherd, "The size, shape and dimension of urban settlements," Transactions of the Institute of British Geographers, vol. 16, no. 1, pp. 75-94, 1991.

[34] R. S. Naroll and L. V. Bertalanffy, "The principle of allometry in biology and social sciences," in General Systems Yearbook, vol. 1, part II, pp. 76-89, 1956.

[35] W. R. Tobler, "Satellite confirmation of settlement size coefficients," Area, vol. 1, no. 3, pp. 30-34, 1969.

[36] L. M. A. Bettencourt, "The origins of scaling in cities," Science, vol. 340, no. 6139, pp. 1438-1441, 2013.

[37] L. M. A. Bettencourt, J. Lobo, D. Helbing, C. Kühnert, and G. B. West, "Growth, innovation, scaling, and the pace of life in cities," Proceedings of the National Academy of Sciences of the United States of America, vol. 104, no. 17, pp. 7301-7306, 2007. 
[38] Y. G. Chen and Y. X. Zhou, "A study of fractional dimension on the relationship between population sizes and economic outputs of cities," Economic Geography, vol. 23, no. 4, pp. 476481, 2003, (Chinese).

[39] L. von Bertalanffy, General System Theory: Foundations, Development, and Applications, George Braziller, New York, NY, USA, 1968.

[40] H. Takayasu, Fractals in the Physical Sciences, Manchester University Press, Manchester, UK, 1990.

[41] M. Batty, The New Science of Cities, The MIT Press, Cambridge, Mass, USA, 2013.

[42] P. J. Taylor, Quantitative Methods in Geography, Waveland Press, Prospect Heights, Ill, USA, 1983.

[43] National Bureau of Statistics of the People's Republic of China, China Statistical Yearbook, Statistics Press, Beijing, China, 2013, (Chinese). 


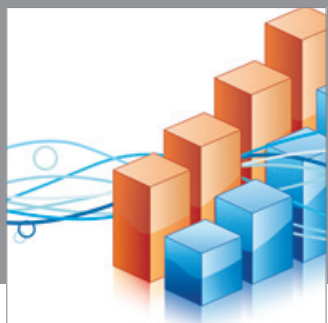

Advances in

Operations Research

mansans

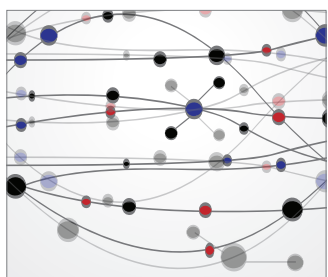

The Scientific World Journal
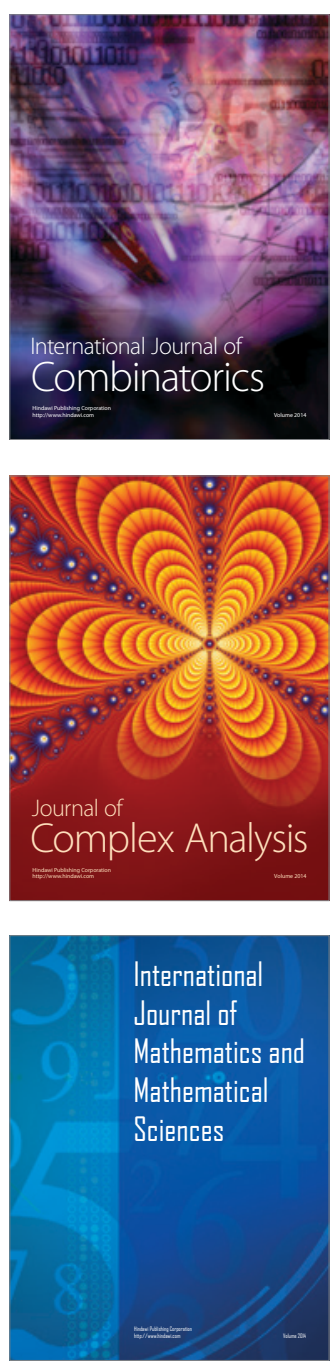
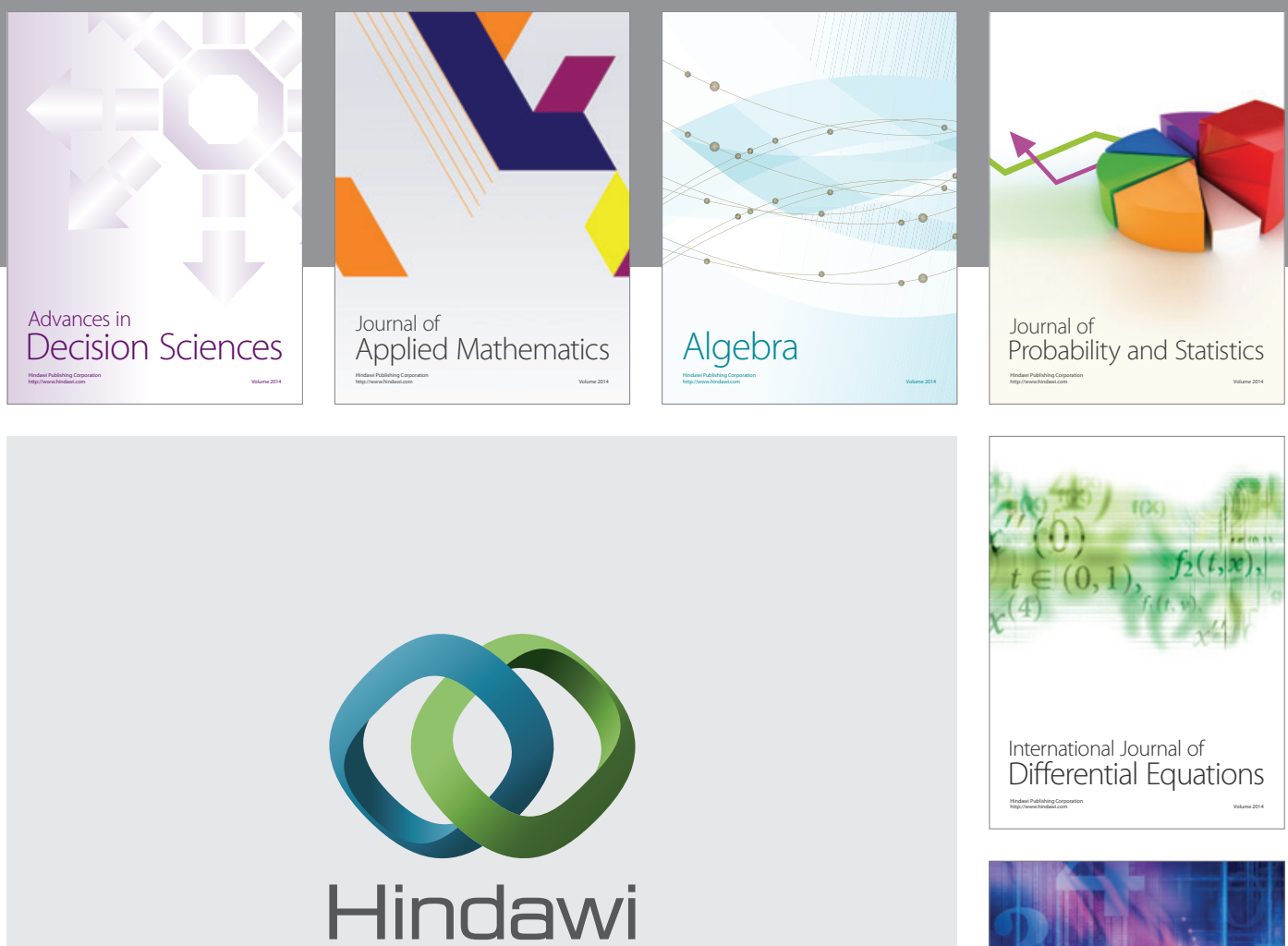

Submit your manuscripts at http://www.hindawi.com
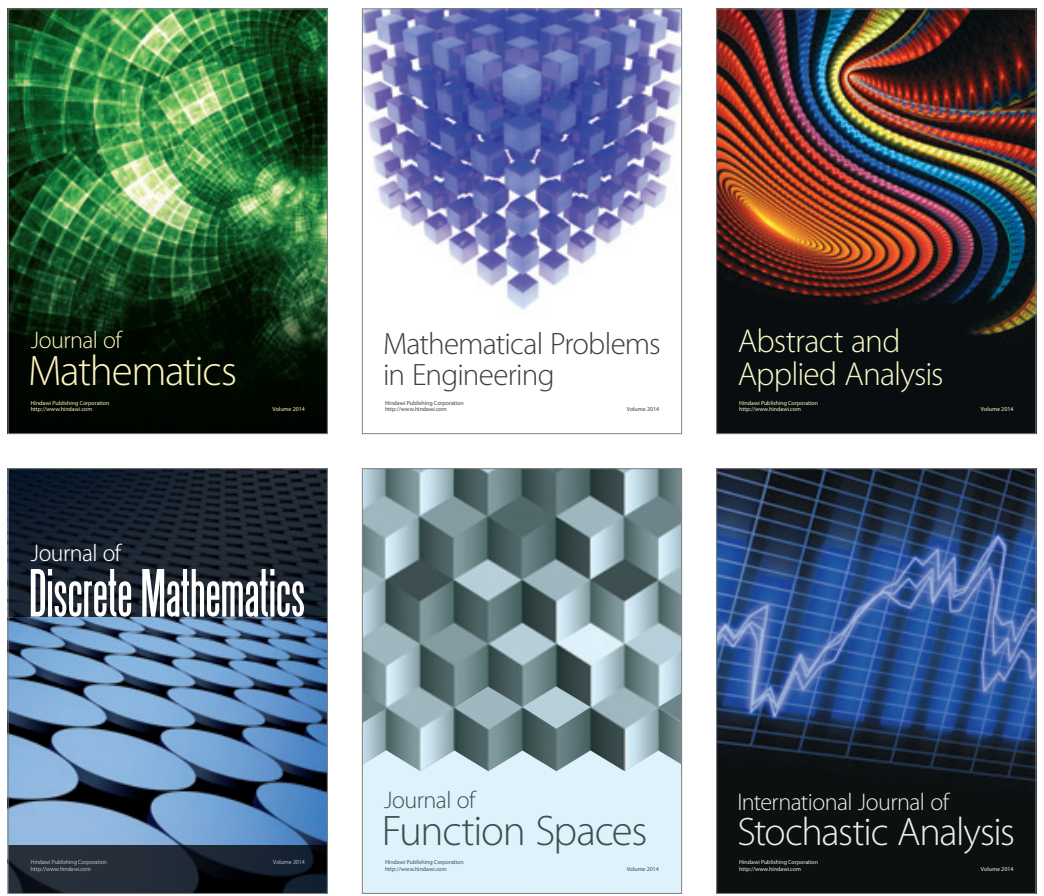

Journal of

Function Spaces

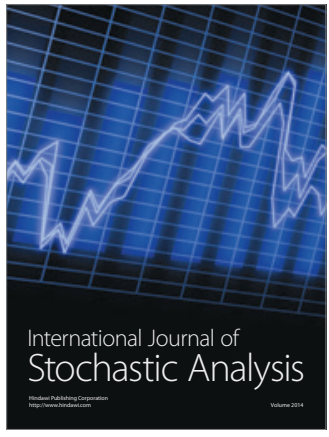

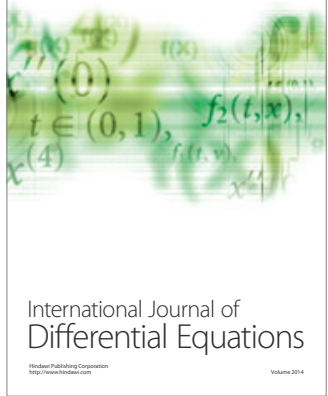
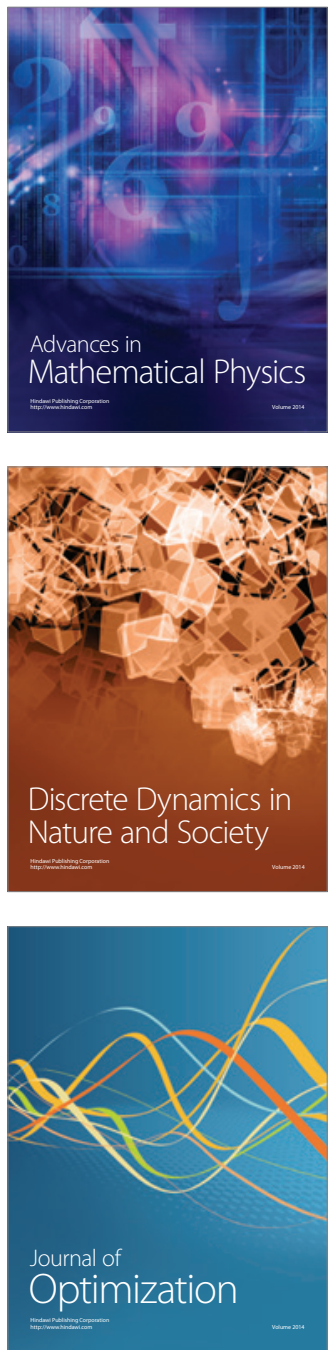